\title{
Antimicrobial Prophylaxis for Caesarean Delivery; A comparative study between different agents
}

\author{
Afroditi Ziogou', loannis Kokolakis², Fanourios Makrygiannakis², Antonis Makrigiannakis² \\ 'Attikon University Hospital, Athens, Greece \\ 'University Hospital of Heraklion, Department of Obstetrics and Gynecology, University Hospital of Heraklion, \\ Heraklion, Greece
}

Corresponding Author

loannis Kokolakis, e-mail: kokolakisioannis@gmail.com

\begin{abstract}
Antimicrobial prophylaxis is commonly used for pre-intra and post-operative caesarean delivery. Caesarean delivery is still the single most important risk factor for puerperal infection. Post-caesarean infections include wound infections, endomyometritis, bacteraemia, septic shock, septic pelvic vein thrombophlebitis, necrotising fasciitis, pelvic abscess, dehiscence of the wound or evisceration. The goal of antimicrobial prophylaxis is to put a stop to postoperative infection of the surgical site, the use of therapeutic antibiotics, additional surgical interventions, longer duration of hospital stay, to decrease postoperative infectious morbidity and mortality and the cost of postoperative health care. A post-caesarean wound infection detected prior to hospital discharge will lead to prolongation of hospital stay and will increase the hospitalisation costs and need of readmission. Both the American College of Obstetricians and Gynaecologists and the American Society of Health-Care System Pharmacists have introduced single-dose prophylactic protocols using a heterogeneity of agents (penicillins, cephalosporins, clindamycin and azithromycin). Women undergoing caesarean delivery have a greater risk of developing infection compared to women who have a vaginal birth by 5 to 20 -fold. The use of prophylaxis in patients undergoing a low-risk Caesarean Delivery remains controversial.
\end{abstract}

Key words: Antibiotics, prophylaxis, caesarean section

\section{Introduction}

Caesarean delivery is the most commonly performed obstetric procedure. Current data also demonstrate that primary caesareans in the absence of obstetric indications are briskly rising reflecting both shifting obstetric practices and maternal preference ${ }^{1}$.
If these tendencies continue, caesareans will make up approximately $50 \%$ of more than 4.000 .000 annual deliveries by 2020. Caesarean delivery is one of the most important risk factors for puerperal infection, ranging from $2.5 \%$ to $20.5 \%$ globally ${ }^{2}$. The aim of 
antibiotic prophylaxis is to prevent postoperative infection of the surgical site and reduce postoperative infectious morbidity and mortality, thus decreasing the duration and the cost of postoperative health care. Postoperative infections include wound infections and endomyometritis, pelvic abscess, bacteraemia, septic shock, septic pelvic vein thrombophlebitis, necrotising fasciitis, dehiscence of the wound or evisceration ${ }^{3}$. Caesarean delivery is the most important risk factor for postpartum endomyometritis with reported odds ratios ranging between 5 and $20^{4}$. Wound infection refers to infection of the skin and subcutaneous tissue at the surgical site. Wound infection found before hospital discharge will lead to further hospital stay and will expand the use of therapeutic antibiotics, additional surgical interventions, the hospitalisation costs and readmission ${ }^{5}$. Common use of prophylactic antibiotics decrease the risk of post-caesarean fever and infections by over $50 \%$ from baseline rates as high as $20-50 \%$ applied to both non-elective and elective procedures ${ }^{6}$. Antibiotic prophylaxis reduces overall length of hospitalisation and decreases treatment cost associated with caesarean, and as a result it is highly cost-effective ${ }^{5}$. Because of the rising rates of caesarean delivery, prevention of post-caesarean infection remains a public health issue priority. Despite the use of currently introduced antibiotic prophylaxis protocols, at least $10 \%$ of caesareans overall are complicated by infection and over $15 \%$ by fever 6 . Without use of of antibiotic prophylaxis approximately $30 \%$ to $40 \%$ of patients who have an urgent caesarean delivery developed endomyometritis; controversially after a scheduled caesarean delivery nearly $10 \%$ to $15 \%$ of patients became infected ${ }^{8}$. The incidence of postpartum endomyometritis is low ( 1-3\%) after vaginal delivery and after spontaneous, complete or missed abortions ${ }^{8}$.

\section{Risk factors}

Post caesarean infections are associated with obesity, diabetes, chorioamnionitis, rupture of membranes $>18$ hours, corticosteroid use, staple suture wound closure, fewer prenatal care visits, emergency caesarean section, length of surgery $>60$ min, prolonged labour, excessive blood loss during labour, immunosuppressive disorders ${ }^{9}$. Among many risk factors for infections an emergency procedure is the most important. Caesarean delivery allows bacteria in the amniotic fluid to contaminate the myometrium, uterine blood vessels and lymphatics at time of hysterotomy. The placenta in a caesarean delivery is preferred be derivered by traction on the umbilical cord rather than by manual extraction. The incidence of endomyometritis ranges from $5 \%$ to $85 \%$ and increases when caesarean delivery is performed after the labour process begins or membranes rupture ${ }^{4}$. Other risk factors include bacterial vaginosis, multiple vaginal examinations in labour, invasive monitoring, pre-existing operative site infection, breaks in sterile technique, use of electrocautery, advanced maternal age, urinary tract infection, development of subcutaneous haematoma, the skill of the operator, method of placental removal and site of uterine repair, as well as poor hygiene and anaemia ${ }^{10}$. Patients undergoing an extended procedure ( 3 hours) or with a total blood loss > $1.500 \mathrm{ml}$ must receive a second dose of antibiotic. Studies have revealed that prophylactic antibiotics decrease the rate of post-caesarean and post abortal endomyometritis as well as post hysterectomy pelvic cellulitis by as much as $50 \%$ to $60 \%$. Obese women with a BMI $>30 \mathrm{~kg} / \mathrm{m}^{2}$ should receive an increased dose of antibiotics prophylaxis ${ }^{11,31,32}$.

\section{Microbiology}

The organisms responsible for obstetric infections fall into two broad categories: sexual transmitted organisms and microorganisms of the endogenous vaginal flora that can be transferred into the upper genital tract with the mechanisms of labour 
and instrumentation during operation. The main pathogens are aerobic Gram-positive cocci (Group B Streptococci, Enterococci, and Staphylococcal species), anaerobic Gram-positive cocci (Peptococci and Peptostreptococci species), aerobic Gram-negative bacilli (E. Coli, Klebsiella pneumonia and Proteus species), and anaerobic Gram-negative bacilli (Bacteroides and Prevotella species). The normal ratio of anaerobes to aerobes is between 2:1 and 5:1. The most frequent organisms isolated from wound infections also include Ureaplasma as well as Staphylococci and Enterococci ${ }^{12}$. Ureaplasma or Mycoplasma is the most prevalent organism isolated from the amniotic fluid at caesarean delivery, and is linked with a 3 to 8-fold increased risk of post-caesarean endomyometritis or wound infection ${ }^{13}$. Bacterial vaginosis is a complicated alteration in the vaginal flora resulting in a high concentration of potentially pathogenic anaerobic bacteria (such as Gardnerella vaginalis, Mycoplasma hominis, Mobilluncus and Bacteroides species), increasing the anaerobic/ aerobic ratio to $100-1.000 / 1$ and multiplying the risk of postcaesarean endomyometritis. Bacterial vaginosis is also correlated with as much as a 6-fold increased risk of post-caesarean endomyometritis ${ }^{14}$. Postpartum endomyometritis is one of the most common infectious complications, which can follow an aggressive course, progressing to pelvic abscess or even generalised peritonitis and septicaemia.

Microorganisms produce toxins and other virulence factors that increase their ability to invade and cause damage to host tissue. Many Gram-negative bacteria produce endotoxins, thereby stimulating massive cytokine production. Some microorganisms have polysaccharide capsules which can inhibit leukocyte phagocytosis, a critical and early host defence response to microbial contamination. Clostridia and Streptococci produce exotoxins that are capable of disrupting cell membranes and/ or altering cellular metabolism ${ }^{15}$. Gram-positive bacteria such as coagulase-negative Staphylococci produce glugocalyx; an associated component referred to as "slime" that physically shields bacteria from phagocytes and inhibits binding and/or penetration of antimicrobial agents ${ }^{16}$. During labour the pathogenic bacteria can ascend into the urinary cavity or colonise the decidual lining and invade the myometrium, setting the stage for postoperative endometritis. Bacteria that invade the uterine cavity can colonize and infect the amniotic fluid and amniotic membranes causing chorioamnionitis. Once chorioamnionitis is established, an infection can progress to cause fetal infection. Postpartum endomyometritis that occurs within the first 48 hours most likely is unimicrobial with Streptococcus Agalactiae or E. Coli being the etiologic agents. Postpartum endomyometritis that occurs later in the postpartum period (greater than 72 hours) is most likely polymicrobial involving facultative and obligate anaerobic bacteria ${ }^{16}$.

\section{Antimicrobial prophylaxis in general}

Concerning about post-caesarean section infection as a cause of direct maternal morbidity and mortality, the specific questions regarding the best evidence for antibiotic prophylaxis, the class of antibiotic, the dose, the timing and the route of administration must be answered. The route of administration of antibiotic prophylaxis must be effective, safe, inexpensive, and convenient. Intravenous administration provides the quickest road to achieve therapeutic plasma levels and has an onset of action of 15 to 30 seconds. The onset of action for the intramuscular route is 10 to 20 minutes. The use of an extended spectrum therapy involving a second antibiotic is not an entirely new concept in surgical antibiotic prophylaxis. In patients with known MRSA colonisation undergoing caesarean, an additional single dose of Vancomycin may be administered. Vancomycin alone is not sufficient for caesarean. For preterm premature rupture of membranes Azithromycin alone or combined to first- 
generation cephalosporins may be an alternative ${ }^{17}$. Using 500mg Azithromycin infused over 1 hour, may consist an alternative solution for women undergoing non elective caesarean. For long procedure greater than 2 drug half-lives administer additional intraoperative dose of the same antibiotic. Amoxicillin/ Clavoulanic acid is not recommended due to increased risk of necrotising enterocolitis ${ }^{18}$. Additionally, a recent cohort study demonstrated a corresponding drop in post-caesarean endomyometritis with increasing use of Azithromycin over a period of 14 years ${ }^{19}$. The incidence of wound infection also reduced from 3.2 to $1.3 \%$ over the same time period ${ }^{20}$.

\section{Comparison among a variety of agents used as antimicrobial prophylaxis in caesarean delivery}

Thirty-five trials of present literature (with 31 trials including 7697 women) were reviewed, comparing safety and effectiveness of different antibiotic prophylactic protocols in women undergoing caesarean section. The trials included women undergoing elective or non-elective caesarean section. Trials were conducted in both low-middle and high income countries. In five trials prophylactic antibiotics were administered after umbilical cord clumping. In four trials antibacterial prophylaxis was administered preoperatively. In the larger part of the trials, cephalosporins were compared to penicillins as prophylaxis to caesarean delivery. Three trials compared a cephalosporin or penicillin with another class of antibiotics, some trials compared single cephalosporin versus single penicillin, other trials compared single cephalosporin versus penicillin combination, cephalosporin combination versus single penicillin and cephalosporin combination versus penicillin combination. Only a few studies compared mixed antibiotic prophylaxis (which does not include cephalosporin or penicillin) with cephalosporin or penicillin.
Thirteen trials including 4010 women compared a cephalosporin versus penicillin as chemoprophylaxis in women delivering with caesarean section ${ }^{21}$. There were no significant differences between groups for post-caesarean endomyometritis (RR- relative risk $1.11,96 \%$ CI to $1.52 ; 9$ trials, 3130 women), maternal febrile morbidity (RR $0.89,95 \%$ CI 0.61 to $1.30 ; 7$ trials, 1344 women) or urinary tract infection (RR $1.48,95 \%$ CI 0.89 to $2.48,7$ trials, 1120 women). There were no clear differences between groups concerning maternal length of hospital stay. No data was reported regarding the number of women readmitted to hospital. There were no important differences between antibiotic groups about adverse effects of the treatment in the women and no allergic reactions to drugs were mentioned in three studies (RR 2.02, 0.5 CI 0.18 to 21.96). There were no cases of severe infectious complications such as maternal sepsis in 346 women in two trials. For the infants, there was no data reported for oral thrush, infant length of hospital stay or immediate adverse effects of the antibiotic. No cases of neonatal sepsis were reported.

Twelve trials (2075 women) compared the administration of cephalosporin with penicillin combination ${ }^{22}$. There were no clear differences between groups for maternal sepsis (RR $2.3795 \%$ CI 0.10 to $56.41 ; 1$ trial, 75 women) post-caesarean endomyometritis (RR 0.90, 95\% CI 0.60 to 1.35; 10 trials, 2134 women), wound infection (RR $0.72,95 \%$ CI 0.40 to 1.30; 7 trials, 1608 women) or urinary tract infection (RR 0.66, 95\% CI 0.17 to 2.55; 6 trials, 1361 women). The outcome of infant sepsis was not mentioned in the included studies.

There were no differences between the use of cephalosporin combination versus single penicillin ( 1 trial, 147 women). No differences were reported in this study concerning post-caesarean endomyometritis (RR 2.70, 95\% CI 0.63 to 11.55), maternal febrile morbidity (RR 2.36, 95\% CI 0.84 to 6.62) 
or wound infection (RR 2.02, 95\% CI 0.42 to 9.63 ). No cases of serious infectious complications were detected.

Two trials (363 women) compared the administration of cephalosporin combination versus penicillin alone. There were no clear differences between groups in terms of this review's maternal primary outcomes: post-caesarean endomyometritis (RR 0.33 , 95\% CI 0.01 to $7.77 ; 1$ trial, 83 women), postpartum febrile morbidity (RR 1.57, 95\% CI 0.69 to $3.60 ; 2$ trials, 315 women), or wound infection (RR 1.23, 95\% CI 0.42 to 3.52 ; 2 trials, 315 women).

\section{Other parameters studied among groups} which were administered antimicrobial prophylaxis

\section{Type of caesarean delivery}

Most of these studies included women undergoing urgent or elective caesarean section. There were no clear differences between groups (women undergoing elective or emergency caesarean delivery) for maternal sepsis (RR 2.91, 95\% CI 0.47 to 18.10; 4 trials, 653 women). However, penicillins demonstrated a higher effectiveness in comparison to cephalosporins in women having an emergency caesarean delivery (RR 1.33, 95\% CI 1.01 to 1.75; 6 trials, 2362 women). There were no important differences between groups of women undergoing elective caesarean delivery (RR 2.06, 95\% CI 0.66 to $6.39 ; 3$ trials, 461 women) ${ }^{23,24}$.

\section{Timing of administration of prophylaxis}

Another parameter studied was the timing of administration of prophylaxis ( 22 trials, 5788 women). The usual practice was to administer antibiotics immediately after cord clumping so as to prevent the passage of the antibiotic into the baby and the suppression of its natural microbioma. This could hide infection in the neonate and promote the selection of resistant organisms ${ }^{25}$. The CDC and the
American Society of Hospital Pharmacists recommend that antibiotic prophylaxis is more effective if administered just prior to surgery ${ }^{26}$, within 60 minutes before the start of the caesarean section. If this is not possible we can administer as soon as possible after the incision ${ }^{27}$. Although ACOG currently does not make a clear recommendation regarding timing, it recognises that prophylactic antibiotics for caesarean section are routinely used following cord clumping ${ }^{25}$. The most recent Cochrane systematic review recommended prophylaxis to be administered after clumping of cord, while other studies suggested pre-incision administration of antibiotics ${ }^{23}$.

\section{Intravenous versus oral \& spraying antibiotic prophylaxis}

In nine studies (included 1274 women) the administration of intravenous antibiotics was compared with antibiotic spraying as prophylaxis in women undergoing caesarean delivery whether elective or non-elective. There were no important differences between comparison groups for postpartum endomyometritis (RR $0.95,95 \%$ CI 0.70 to $1.29,8$ studies, 966 women) and wound infection (RR 0.49 , $95 \%$ CI 0.17 to $1.45,7$ studies, 859 women). None of the included studies reported infant sepsis. There were no significant differences between comparison groups regarding postpartum febrile morbidity (RR 0.87, 95\% CI 0.48 to $1.60,3$ studies, 264 women), maternal urinary tract infection (RR $0.74,95 \%$ CI 0.25 to 2.15 ; 5 studies, 660 women), while there were no cases of severe infectious complications. There were no significant differences between groups concerning maternal length of hospital stay and number of women readmitted to hospital ${ }^{9,28}$.

One study compared an intravenous with an oral route of administration of prophylactic antibiotics but did not demonstrated any of this review's primary or secondary outcomes ${ }^{9}$.

There were no clear differences between com- 
parison groups about timing of administration for maternal sepsis (RR 2.91, 95\% CI 0.47 to $18.10 ; 4$ studies, 653 women), or postpartum endomyometritis (RR 1.11, 95\% CI 0.90 to 1.37; 20 trials, 5390 women).

\section{Comparison between different types of antibiotics}

First generation cephalosporins are highly recommended than broader spectrum antibiotics; they are equally effective, safe and less over-priced than the next generations ${ }^{29}$. Two trials (822 women) compared the administration of a first-generation cephalosporin versus extended spectrum penicillins. There were no significant differences between groups for postpartum morbidity due to fever (RR $2.36,95 \%$ CI 0.84 to 6.62 ; 1 trial, 139 women), or wound infection (RR 2.02, 95\% CI 0.42 to $9.63 ; 1$ trial, 139 women) but extended-spectrum penicillin were more efficient in preventing post-caesarean endomyometritis (RR 2.18, 95\% CI 1.30 to 3.66 ; 2 trials, 814 women $)^{30}$.

In eight studies (1882 women) the administration of first-generation cephalosporins was compared with aminopenicillins. There were no clear differences between groups regarding post-caesarean endomyometritis (RR 1.09, 95\% CI 0.69 to $1.71 ; 7$ studies, 1487 women), postpartum febrile morbidity (RR $0.78,95 \%$ CI 0.40 to $1.51 ; 5$ studies, 626 women), wound infection (RR $0.85,95 \%$ CI 0.36 to $2.01 ; 5$ studies, 626 women) or urinary tract infection (RR $1.41,95 \%$ CI 0.54 to 3.70 ; 5 studies, 626 women). A decrease was observed regarding maternal length of hospital stay in women who received first-generation cephalosporins to aminopenicillins (MD-1.50 days, $95 \%$ CI 2.46 to $0.54 ; 1$ study, 132 women) ${ }^{21,30}$.

In six studies (2,077 women) the administration of second-generation cephalosporins was compared versus extended spectrum penicillins. There were no differences between groups for post caesarean endomyometritis (RR 1.10, 95\% CI 0.78 to $1.54 ; 4$ studies, 1,334 women), postpartum febrile morbidity (RR 1.08, 95\% CI 0.79 to $1.47 ; 4$ studies, 850 women), urinary tract infection (RR 1.43, 95\% CI 0.67 to $3.07 ; 3$ studies, 567 women) or wound infection (RR 2.37, 95\% CI 0.64 to $8.73 ; 2$ studies, 438 women). None drug allergic reactions were reported in two studies (1,030 women). No cases of serious infectious complications were reported. There was no clear difference between groups in maternal length of hospital stay and readmissions to hospital ${ }^{21,22,30}$.

In eight studies (1,921 women) second-generation cephalosporins were compared versus aminopenicillins. There were no important differences between groups in postpartum endomyometritis (RR 1.10, 95\% CI 0.75 to 1.35 ; 8 studies, 1,890 women), postpartum morbidity due to fever (RR 1.17, 95\% CI 0.64 to $2.15 ; 3$ studies, 387 women), wound infection (RR $1.14,95 \%$ CI 0.47 to 2.78 ; 5 studies, 638 women) or urinary tract infection (RR $0.63,95 \%$ CI 0.11 to 3.66 ; 4 studies, 462 women). Infections can be induced by surgical incision, the lining of the uterus and inside the pelvis. The signs/ symptoms of post partum endomyometritis usually occur within 5 days after delivery ${ }^{16}$. Caesarean section enlarges the risk of infection compared to vaginal by 5 to 20 -fold ${ }^{21,22,30}$.

Two studies (359 women) compared the administration of intravenous third-generation cephalosporins versus extended spectrum penicillin. Extended spectrum penicillin was more essential than thirdgeneration cephalosporins in preventing postpartum endomyometritis (RR 2.14, 95\% CI 1.14 to 4.00 ; 1 study, 300 women $)^{22,23}$.

In seven studies (1,904 women) the administration of third-generation cephalosporins was compared versus aminopenicillins. There were no evident differences between groups for postpartum endomyometritis (RR 1.47, 95\% CI 0.89 to 2.42 ; 5 studies, 1,472 women), postpartum febrile morbidity (RR 
$1.12,95 \%$ CI 0.69 to $1.83 ; 3$ studies, 1,060 women), urinary tract infection (RR $0.52,95 \%$ CI 0.10 to 2.80; 2 studies, 233 women), or maternal length of hospital stay (MD- $0.03,95 \%$ CI 0.14 to 0.08 ; 1 study, 746 women). No data was reported concerning the number of women readmitted to hospital. Wound infections were decreased in the group receiving third-generation cephalosporins (RR 0.49, 95\% CI 0.27 to $0.90 ; 6$ studies, 1,556 women) ${ }^{22,23,30}$.

One study (72 women) compared ciprofloxacin receiving versus Ampicillin/ Sulbactam and another trial (81 women) compared ciprofloxacin to cefotetan. There were no clear differences between groups regarding postpartum endomyometritis, wound infection, postpartum morbidity due to fever or severe infectious complications ${ }^{21,22}$.

Three small studies compared the Lincosamide plus aminoglycoside versus Penicillin (1 study, 88 women), and 2 studies (118 women) compared the beta-lactam antibiotics versus cephalosporins. There were no significant differences between groups about postpartum endomyometritis, wound infection, maternal sepsis or postpartum febrile morbidity ${ }^{22,30}$.

One study (241 women), compared Gentamycin plus Metronidazole versus a cocktail of antibiotics (Penicillin, Nitroimidazole and Macrolide). There were no important differences between groups regarding postpartum endomyometritis (RR 0.81, $95 \%$ CI 0.29 to 2.26 ; 1 study, 241 women), postpartum febrile morbidity (RR $1.12,95 \%$ CI 0.69 to $1.83,3$ studies, 1.060 women), wound infection (RR $3.23,95 \%$ CI 0.34 to 30.64 ; 1 study, 241 women), or maternal urinary tract infection (RR 1.08, 95\%CI 0.07 to 17.03 ; 1 study, 241 women). There were differences between groups in terms of maternal length of hospital stay, or about the number of readmissions to hospital. The outcome of infant sepsis was not demonstrated in the subsequent studies $^{22,30}$.

\section{References}

1. MacDorman MF, Menaker F, Declercq E. Cesarean birth in the United State: epidemiology, trends and outcomes. Clin Perinatol 2008;35(2):293-307.

2. Conroy K, Koenig AE, Yu YH, Courney A, Lee HJ, Norwich ER. Infectious morbidity after caesarean delivery: 10 strategies to reduce risk. Reviews in Obstetrics and Gynecology 2012; 5 (2) 69-77.

3. Lamont RF, Sabel JD, Kusanonic JP, Vaisbuch E, Mazaki-ToviS, Kim SK et al. Current debate on the use of antibiotic prophylaxis for caesarean section. BJOG: an international journal of obstetrics and Gynecology 2011; 118: 193-201.

4. Olsen MA, Burler AM, Willers DM, Gross GA, Deucota P, Fraser YJ: Risk factors for endometritis after low transverse caesarean delivery. Control and Hospital Epidemiology 2010; 31: 69-77.

5. Griffiths J, Demianczuk N, Cordoviz M, Joffe AM. Surgical site infection following elective caesarean section: a case control study of postdischarge surveillance. Journal of Obstetric and Gynaecology Canada 2005;27:340-4.

6. Smaill F, Hofmeyer GJ. Antibiotic prophylaxis for caesarean section. Cochrane Database of systemic Reviews, 2002;(3).

7. Chelmow D, Hennesy M, Evantash EG. Prophylactic antibiotics for non-laboring patients with intact membranes undergoing caesarean delivery: an economic analysis. Am J Obstet Gynecol 2004; 181 (5): 1661-5.

8. Leth RA, Moller JK, Thomsen RW, Uldbjerg N, NorgaaldM. Risk of selected postpartum infections after caesarean section compared with vaginal birth: a five year cohort study of 32.468 women. ActoObstetricia et Gynecologica Scandinavica 2009; 88: 976-83.

9. Nabhan AF, Allam NE, Hamed Abdel-Aziz Salama M. Routes of administration of antibiotic prophylaxis for preventing infectio after caesarean section. Cochrane Database of systematic reviews 
2016, Issue 6.

10. Maharaj D. Puerperal pyrexia: a review. Part 1. Obstetrical et Gynaecological survey 2007; 62:393-9.

11. Sullivan AS, Smith T, Chang E, et all. Administration of cefazolin prior to skin incision at cord clamp in preventing postcesarean infectious morbidity: a randomized controlled trial. Am J Obstst Gynecol, 2007; 196:431-455.

12. Oluwatosin Jaiyeoba. Postoperative infections in obstetrics and gynaecology. Clinical Obstetrics and Gynecology vol 25, Nr 4, 904-913, 2012.

13. Alan TN Tita, Dwight J Rouse, Sean Blackwell, George R Saade, Catherine Y Spong and William W Andrews. Evolving concepts in Antibiotic Prophylaxis for caesarean delivery: A systematic review. Obstet Gynecol 2009 March; 113(3): 675-682.

14. Chain W, Bashiri A, Bar- David J, et all. Prevalence and clinical significance of postpartum endometritis and wound infection. Infect Dis Obstet Gynecol 2000; 9: 77-82.

15. Faro S. Postpartum endometritis. Clin Perinatal 2005; 32(3): 803-14.

16. Faro C, Faro S. Postoperative pelvic infections. Infect Dis Clin N Am 22(2008) 653-663.

17. Kenyon S, Boulvain M, Neilson J. Antibiotics for preterm rupture of the membrane: a systematic review. Obstet Gynecol 2004; 104(5pt1): 1051-7.

18. ACOG Guidance: Antibiotic prophylaxis during labor and delivery. https:www.obgproject. com/2018/08/29.

19. Tita AT, Hauth JC, Grimes A, Owen J, Stamm AM, Andrews WW. Decreasing incidence of post-cesarean endometritis with extended-spectrum antibiotic prophylaxis. Obstet Gynecol 2008; 111 (1):51-6.

20. Tita AT, Owen J, Stamm AM, Grimes A, Hauth JC, Andrews WW. Impact of Extended-spectrum Antibiotic prophylaxis on Incidence of post caesarean surgical wound infection. Am J Obstet Gynecol 2008;199(3):303, el-3.

21. WHO recommendation on antibiotic prophylaxis for caesarean section using a single dose of first generation cephalosporin or penicillin in preference to other classes of antibiotics 01 September 2015 .

22. Gyte GM, Dou L, Vazquez JC. Different classes of antibiotics given to women routinely for preventing infection at caesarean section. The Cochrane database of systematic reviews. 2014(11):Cd008726.

23. Smail FM, Grivell RM. Antibiotic prophylaxis versus no prophylaxis for preventing infection after caesarean section. Cochrane Database of systematic reviews 2014, Issue 10.

24. Tita AT, Rouse DJ, Blackwell S, Saade GR, Spong $\mathrm{CY}$, Andrews WW. Emerging concepts in antibiotic prophylaxis for caesarean delivery: a systematic review. Obstet and Gynecol 2009;113:675-82.

25. Cantwell R, Clutton- Brock T, Cooper G, Dowson A, Drife J, Garrod D, et all. Saving mothers lives. Reviewing maternal deaths to make motherhood safer: H 2006-2008. The eight report of the confidential enquities into maternal deaths in the United Kingdom. BJOG: an international journal of obstetrics and gynecology 2011; 118 Suppl1:1-203.

26. Chelmow D, Hennesy M. Evantash EG. Prophylactic antibiotics from non-laboring patients with intact membranes undergoing caesarean delivery:an economic analysis. Am J Obstet Gynecol 2004;191(5):1661-5.

27. Leth RA, Moller JK, Thomsen RW, Uldberg N Norgaald M. Risk of selected postpartum infections after caesarean section compared with vaginal birth:a five year cohort study of 32.468 women. Acta Obstetrica et Gynecologica Scandinavica 2009;88:976-83.

28. Mackeen AD, Packard RE, Ota E, Berhella V, Baxter JK. Timing of intravenous prophylactic antibiotics for preventing postpartum infectious morbidity in women undergoing caesarean delivery. Cochrane Database of systematic reviews 2014, Issue 12.

29. American College of Obstetricians and Gynecolo- 
gist. ACOG practice bulletin nr 47, October 2003: Prophylactic Antibiotics in Labor and Delivery. Obstet Gynecol 2003;102(4):875-82.

30. Alfirevic Z, Gyte GML, Dou L. Different classes of antibiotics given to women routinely for preventing infection at caesarean section. Cochrane Database of systematic reviews 2010, Issue 10 .

31. Myles TD, Gooch J, Santolaya J. Obesity as an independent risk factor for infectious morbidity in patients who undergo caesarean delivery. Obstet- rics and Gynecology 2002;100:959-64.

32. Olsen MA, Bugler AM, Willers DM, Devkota P, Gross GA, Fraser VJ. Risk factors for surgical site infection after low transverse caesarean section. Infection Control and Hospital Epidemiology 2008;29:47784; discussion 485-5.

Received 02-12-20

Revised 10-12-20

Accepted 14-12-20 